\title{
Evaluation of the Tantalum-Titanium phase diagram from ab-initio
}

\section{calculations}

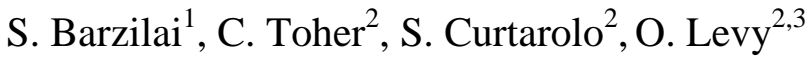 \\ ${ }^{1}$ Department of Materials Science, NRCN, P.O.Box 9001, Beer-Sheva 84190, Israel. \\ ${ }^{2}$ Department of Mechanical Engineering and Materials Science, Duke University, Durham, North Carolina \\ 27708, USA \\ ${ }^{3}$ Department of Physics, NRCN, P.O.Box 9001, Beer-Sheva 84190, Israel.
}

\section{Abstract}

The thermodynamic properties of the Ta-Ti binary system below $900^{\circ} \mathrm{C}$ are not well known. In particular, the location and shape of the solvus between the phase separation region at low temperatures and the solid solution at high temperatures are not well defined. In this study, we present a thermodynamic description for this system based on ab-initio calculations. The formation enthalpies of bcc and hcp solid solutions are estimated using the special quasi-random structures methodology and their vibrational free energy calculated by the quasi-harmonic Debye model. The excess energies of the solid solutions are fitted to a sub-subregular model and used to define the phase diagram of the binary system. It is shown that the current empirical assessment of the energies of the pure elements leads to a phase diagram that strongly departs from the known experimental features at low temperatures. An ab-initio guided correction of these energies is necessary to obtain correctly the low temperature phase separation and the high temperature solid solution. The predicted solvus of the phase diagram is qualitatively different from those previously reported for the Ta-Ti system. It exhibits a miscibility gap between two distinct bec phases, similar to those that exist in the closely related binary systems Ta-Zr, Ta-Hf, Cr-Ti, Mo-Ti, V-Ti, and Ti-W. 


\section{Introduction}

The demand for materials suitable for permanent implants in the human body grows as people live longer and their bones weaken with age. Ta-Ti alloys have been proposed as one of the best choices for biomedical applications based on their excellent biocompatibility in the human body environment, high strength, relatively low elastic moduli and enhanced corrosion resistance $[1,2,3$, $4,5,6]$. These alloys are also attractive for applications in advanced actuators since they exhibit shape memory behavior at relatively high temperatures due to a reversible martensitic transformation between the meta-stable bcc phase and the $\alpha$ " martensite phase $[7,8,9,10,11,12,13]$. Despite the potential applications of these alloys, important gaps exist in our knowledge of their binary phase diagram. The phase diagram has been studied experimentally $[14,15,16,17]$ and modeled using the computational thermodynamics CALPHAD approach $[18,19,20]$. In these studies, no inter-metallic compounds were observed in the Ta-Ti system. At temperatures higher than $400-900^{\circ} \mathrm{C}$, depending on the composition, the stable phase is a single bcc solid solution that decomposes into the elemental hcp and bcc structures at lower temperatures. Fig. 1 shows a schematic Ta-Ti phase diagram assessed from the latest thermodynamic data [20].

Some controversy exists as to the exact location of the solvus $[15,16,17,18]$. The various reports on the locus of the solvus were discussed by Murray [21], who suggested that the variation was probably due to contaminations or inadequate experimental quench rates. Knowledge of the accurate location of the solvus is necessary for the design of thermal treatments for control and tailoring of the properties of Ta-Ti alloys.

To the best of our knowledge there are no published ab-initio studies to compute the Ta-Ti phase diagram. In this study we utilize density functional theory (DFT) to study this system at temperatures below $900^{\circ} \mathrm{C}$. We employ the AFLOW high-throughput framework [22] to screen a database of ordered structures and estimate the formation enthalpies of bcc and hep solid solutions using the special quasi-random structures (SQS) methodology [23]. The ideal expression for the 
configuration entropy and the quasi-harmonic Debye model for the vibrational energy are used to estimate the finite temperature contributions to the free energy. This analysis of the alloys' free energy leads to a binary phase diagram that is qualitatively different from those previously reported for the Ta-Ti system. It exhibits a wide phase separation hump on the solvus between two distinct bcc phases, similar to those that appear in the closely related binary systems $\mathrm{Ta}-\mathrm{Zr}$, Ta-Hf, Ti-Cr, TiMo and Ti-W [24]. It thus improves our thermodynamic understanding of the system and provides a more solid basis for both fundamental and applied research into Ta-Ti alloys. In particular, this understanding should assist the development of better thermal treatments for applications of Ta-Ti products.

\section{Methodology}

\subsection{DFT calculations}

To investigate the Ta-Ti phase diagram we utilized $a b$-initio electronic structure calculations within the framework of density functional theory (DFT). We start by screening an extensive database of ordered stoichiometric structures via the high-throughput framework AFLOW [22]. In addition, the pure elements in the hcp and bcc structures and SQS on the bcc and hcp lattices, at compositions $\mathrm{Ta}_{0.75} \mathrm{Ti}_{0.25}, \mathrm{Ta}_{0.5} \mathrm{Ti}_{0.5}$ and $\mathrm{Ta}_{0.25} \mathrm{Ti}_{0.75}$, are calculated to estimate the formation energies of the corresponding solid solutions. For these calculations, we used the 16-atoms per unit cell structures reported in [25] and [26] for the bcc- and hcp-SQS, respectively. All total energy calculations were carried out employing the VASP software [27] within the AFLOW standard for material structure calculations $[22,28]$. We used projector augmented waves (PAW) pseudopotentials [29] and the exchange correlation functionals parameterized by Perdew, Burke, and Ernzerhof (PBE) [30] for the generalized gradient approximation (GGA). The energies were calculated at zero temperature and pressure, with spin polarization and without zero-point motion or lattice vibrations. All crystal structures were fully relaxed (cell volume and shape and the basis atom coordinates inside the cell). 
Numerical convergence to about $1 \mathrm{meV} /$ atom was ensured by a high-energy cutoff, $30 \%$ higher than the highest energy cutoff for the pseudopotentials of the components, and dense $6000 \mathrm{k}$-point per reciprocal atom Monkhorst-Pack meshes [31]. Complete information about the over 200 ordered structures calculated for the Ta-Ti system, including initial and relaxed structures and detailed calculation specifications, can be obtained in the open access AFLOWLIB materials data repository $[32,33]$.

It should be noted that, under full relaxation, the hcp-SQS $\mathrm{Ta}_{0.75} \mathrm{Ti}_{0.25}$ and $\mathrm{Ta}_{0.5} \mathrm{Ti}_{0.5}$ changed their geometry and relaxed into the corresponding bcc-SQS. This is in qualitative agreement with the experimental phase diagram of Ta-Ti alloys (Fig. 1), where instability of the hcp solid solution should be expected at high Ta concentrations. Therefore, for these structures, we also performed a series of partial relaxations, volume relaxation followed by either shape relaxation for the unit cell and atomic minimization for the location of the atoms in this unit cell or vice versa. The properties of the hcp solid solution at these concentrations are hereafter estimated using the results of these restricted calculations, selecting the lowest energy obtained by a structure that preserved the hcp symmetry under partial relaxations. For the $\mathrm{Ta}_{0.75} \mathrm{Ti}_{0.25}$ hcp-SQS this lowest energy is obtained by a volume relaxation followed by a relaxation of atomic positions without shape relaxation. For the $\mathrm{Ta}_{0.5} \mathrm{Ti}_{0.5}$ it is obtained by a volume relaxation followed by consecutive but separate atom positions and shape relaxations.

The calculations of the total energies of the relaxed hcp- and bcc-SQS were repeated using a full potential method, employing the Augmented Plane Waves + local orbitals (APW+lo) formalism as implemented in the WIEN2k code $[34,35]$. In these calculations the core states treatment is fully relativistic [36] and the valence states are considered in the scalar relativistic approximation [37]. The GGA-PBE exchange correlation potential was employed, as in the VASP calculations. The radii of the muffin-tin spheres $\left(\mathrm{R}_{\mathrm{mt}}\right)$ were 2.3 a.u. for both Ti and Ta. It was found that a basis-set size of $\mathrm{R}_{\mathrm{mt}} \mathrm{K}_{\max }=11$ and a $k$-mesh of 600 points for the SQS and 3500 points for the pure elements suffices 
to reach an accuracy of $\sim 10^{-4} \mathrm{Ry}$ in the total-energy calculations, with an energy cutoff separating core and valence states of $-6 \mathrm{Ry}$. Here $\mathrm{K}_{\max }$ represents the magnitude of the largest $\mathrm{K}$ vector in the wave function expansion. Partial structure relaxation was carried out by optimization of the lattice parameters and minimization of the internal location of the atoms in the cell as described in $[38,39]$.

\subsection{Thermodynamic modeling}

The formation enthalpy of a binary intermetallic structure is given by

(1) $\mathrm{H}^{\mathrm{f}}\left(\mathrm{Ta}_{(1-\mathrm{x})} \mathrm{Ti}_{\mathrm{x}}\right)=\mathrm{H}\left(\mathrm{Ta}_{(1-\mathrm{x})} \mathrm{Ti}_{\mathrm{x}}\right)-(1-\mathrm{x}) \mathrm{H}(\mathrm{Ta})-\mathrm{xH}(\mathrm{Ti})$

where $\mathrm{H}\left(\mathrm{Ta}_{(1-\mathrm{x})} \mathrm{Ti}_{\mathrm{x}}\right)$ is the enthalpy per atom of the intermetallic structure and $\mathrm{H}(\mathrm{Ta})$ and $\mathrm{H}(\mathrm{Ti})$ are the enthalpies per atom of the elements at their ground state structures, bcc-Ta and hcp-Ti. Negative formation enthalpies signify structures that are energetically favorable compared to phase separation into the elemental structures and are therefore possible candidates for stable compounds in the binary system.

The free energies of the bcc and the hcp solid solutions for Ta-Ti alloys $\mathrm{G}\left(\mathrm{x}_{\mathrm{Ta}}, \mathrm{x}_{\mathrm{Ti}}, \mathrm{T}\right)$, are expressed by

(2) $\mathrm{G}^{\varphi}\left(\mathrm{x}_{\mathrm{Ta}}, \mathrm{x}_{\mathrm{Ti}}, \mathrm{T}\right)=\mathrm{x}_{\mathrm{Ta}} \cdot{ }^{0} \mathrm{G}_{\mathrm{Ta}}^{\varphi}(\mathrm{T})+\mathrm{x}_{\mathrm{Ti}} \cdot{ }^{0} \mathrm{G}_{\mathrm{Ti}}^{\varphi}(\mathrm{T})+{ }^{\mathrm{mix}} \mathrm{G}^{\varphi}\left(\mathrm{x}_{\mathrm{Ta}}, \mathrm{x}_{\mathrm{Ti}}, \mathrm{T}\right)+{ }^{\mathrm{ex}} \mathrm{G}^{\varphi}\left(\mathrm{x}_{\mathrm{Ta}}, \mathrm{x}_{\mathrm{Ti}}, \mathrm{T}\right)$

where $\varphi$ represents the bcc or the hcp structures, $T$ is the absolute temperature, and ${ }^{0} \mathrm{G}_{\mathrm{Ta}}$ and ${ }^{0} \mathrm{G}_{\mathrm{Ti}}$ are the Gibbs energies of the pure elements. The configuration energy ${ }^{\text {mix }} G^{\varphi}$ is the contribution of the ideal solution

$$
{ }^{\text {mix }} \mathrm{G}^{\varphi}\left(\mathrm{x}_{\mathrm{Ta}}, \mathrm{x}_{\mathrm{Ti}}, \mathrm{T}\right)=\mathrm{kT}\left(\mathrm{x}_{\mathrm{Ta}} \ln \mathrm{x}_{\mathrm{Ta}}+\mathrm{x}_{\mathrm{Ti}} \ln \mathrm{x}_{\mathrm{Ti}}\right)
$$

and the excess energy

$$
{ }^{\mathrm{ex}} \mathrm{G}^{\varphi}={ }^{\mathrm{ex}} \mathrm{H}^{\varphi}+{ }^{\mathrm{ex}} \mathrm{G}^{\varphi} \text { vib }
$$

with

$$
{ }^{\text {ex }} \mathrm{H}^{\varphi}=\mathrm{H}_{\mathrm{TaTi}}^{\varphi}-\mathrm{X}_{\mathrm{Ta}} \cdot \mathrm{H}_{\mathrm{Ta}}^{\varphi}-\mathrm{X}_{\mathrm{Ti}} \cdot \mathrm{H}_{\mathrm{Ti}}^{\varphi} \text { and }
$$




$$
{ }^{\text {ex }} \mathrm{G}_{v i b}^{\varphi}=\mathrm{G}_{\mathrm{vib}, \mathrm{TaTi}}^{\varphi}-\mathrm{X}_{\mathrm{Ta}} \cdot \mathrm{G}_{\mathrm{vib}, \mathrm{Ta}}^{\varphi}-\mathrm{X}_{\mathrm{Ti}} \cdot \mathrm{G}_{\mathrm{vib}, \mathrm{Ti}}^{\varphi}
$$

represents the effect of non-ideality. The excess enthalpy, ${ }^{e x} \mathrm{H}^{\varphi}$, is computed directly from the DFT results for the corresponding SQS and pure element structures. The vibrational contribution, $\mathrm{G}_{\mathrm{vib}}$, is estimated by the Debye model [40, 41],

(6) $\mathrm{G}_{\mathrm{vib}}=\frac{9}{8} \mathrm{R} \theta_{\mathrm{D}}+\mathrm{RT}\left[3 \ln \left(1-\exp \left(-\frac{\theta_{\mathrm{D}}}{\mathrm{T}}\right)\right)-3 \frac{\mathrm{T}^{3}}{\theta_{\mathrm{D}}^{3}} \int_{0}^{\theta_{\mathrm{D}} / \mathrm{T}} \frac{\mathrm{z}^{3} \mathrm{dz}}{\exp (\mathrm{z})-1}\right]$

where

$$
\theta_{\mathrm{D}}(\mathrm{T})=\frac{\hbar}{\mathrm{k}_{\mathrm{B}}}(6 \pi \sqrt{\mathrm{V}} \mathrm{n})^{\frac{1}{3}} \mathrm{f}(\sigma) \sqrt{\frac{\mathrm{B}_{\mathrm{T}}}{\mathrm{M}}}
$$

is the Debye temperature,

(8) $f(\sigma)=\left\{3\left[2\left(\frac{2}{3} \frac{1+\sigma}{1-2 \sigma}\right)^{3 / 2}+\left(\frac{1}{3} \frac{1+\sigma}{1-\sigma}\right)^{3 / 2}\right]^{-1}\right\}^{1 / 3}$,

$\mathrm{M}$ is the mass of the unit cell, $\mathrm{V}$ is its volume and $\mathrm{n}$ is the number of atoms in it, $\mathrm{B}$ is the bulk modulus and $\sigma$ is the Poisson ratio. The electronic and the magnetic excess contributions to the Gibbs energy are expected to be much smaller in this system and are therefore neglected.

\section{Results}

The high-throughput screening of over 200 structures identified no stable structures in the Ta-Ti system, in agreement with the experimental data that includes no Ta-Ti compounds. The formation enthalpies of the bcc- and hcp- SQS were also found to be positive, in agreement with the known phase separation in the binary system at low-temperatures. Fig. 2 presents the formation enthalpies of various ordered compounds and the bcc- and hcp- SQS, with space group labels denoting the compounds with the lowest formation enthalpies. As mentioned in the introduction, the complete information about all of the structures can be found in the open access AFLOWLIB materials data repository $[32,33]$. 
The Gibbs energies of solid solutions of a specific structure are obtained by Eq. (2) from the energies of the pure elements in the same structure, and the corresponding configuration and excess energies. Here, we describe the excess free energy of the bcc and hcp structures as a function of alloy composition by fitting the excess energies of the elements and the SQS, calculated from Eq. (4), to a sub-subregular model using a Redlich-Kister polynomial of the fourth degree [42]

(9) ${ }^{\mathrm{ex}} \mathrm{G}=\mathrm{x}_{\mathrm{Ta}} \mathrm{x}_{\mathrm{Ti}}\left({ }^{0} \mathrm{~L}_{\mathrm{TaTi}}+{ }^{1} \mathrm{~L}_{\mathrm{TaTi}}\left(\mathrm{x}_{\mathrm{Ta}}-\mathrm{x}_{\mathrm{Ti}}\right)+{ }^{2} \mathrm{~L}_{\mathrm{TaTi}}\left(\mathrm{x}_{\mathrm{Ta}}-\mathrm{x}_{\mathrm{Ti}}\right)^{2}\right)$.

The coefficients ${ }^{0} \mathrm{~L},{ }^{1} \mathrm{~L}$ and ${ }^{2} \mathrm{~L}$, are the Redlich-Kister coefficients that reconstruct the interaction energy between $\mathrm{Ta}$ and $\mathrm{Ti}$ atoms in the corresponding phase.

The DFT results for the energies of the elements and the SQS are shown in Fig. 3 together with the fitted polynomials for the hcp, Fig. 3(a), and the bcc, Fig. 3(b), solid solutions. The DFT results obtained from the VASP and WIEN2k calculations are very close, with an apparent but insignificant discrepancy at the $\mathrm{Ta}_{0.75} \mathrm{Ti}_{0.25}$ bcc-SQS. The fitted sub-subregular model (solid lines) is used to retrieve the Redlich-Kister coefficients and to express the excess free enthalpy for the entire range of compositions in the Ta-Ti system for both lattices. The dashed lines in the figures represent the thermodynamic models evaluated from experimental data for the bcc and the hcp phases of the Ta-Ti system [20]. As can be seen, the excess enthalpy calculated by DFT is qualitatively different from the enthalpy evaluated from experiments [20] that underlies the currently published phase diagram of Ta-Ti. The experimental data shows a repulsive interaction between $\mathrm{Ta}$ and $\mathrm{Ti}$ for the entire components concentration range, in both the bcc and hcp phases. Our calculated results, however, reveal a more complex behavior. Attraction is obtained for the hcp lattices, which is particularly strong at low Ti concentrations, whereas for the bcc lattices a repulsive interaction was obtained for alloys with low Ti concentrations and an attractive interaction for high Ti concentrations. The results obtained for the bcc lattice are similar to those recently obtained for bcc alloys in the U-Ti system [43], where the experimental results indicate a repulsive interaction for all Ti concentrations [44] while DFT calculations reveal a repulsive interaction only at low Ti content. 
To determine the excess Gibbs energy at elevated temperatures, the entropy effects have to be considered. In this study we consider the configuration entropy of an ideal solution and estimate the vibrational entropy according to the GIBBS methodology [45]. It is well known that the evaluation of the thermodynamic properties of materials by the Debye model (Eqs. (6-8)) may produce values that deviate from experimental data $[46,47,48]$. Thus, to estimate the sensitivity of the excess vibration energy to these deviations we considered four sets of Debye temperatures. The bulk moduli of the pure elements and of each of the SQS were computed by the AFLOW-AGL software [48], and the specific atomic volume for each structure retrieved directly from the DFT calculations. They are shown in table 1. For each set of Debye temperatures we considered a different estimate for the Poisson ratio dependent scaling factor, $f(\sigma)$, in Eq. (7). The first set includes the empirical generic scaling factors, 0.617 [46] and 0.7 [47], for the bcc and hep structures respectively. These results for the pure elements and the SQS are also shown in table 1. In the second set we used a uniform Poisson ratio $\sigma=0.25$, corresponding to an ideal Cauchy solid, that gives a scaling factor of 0.86 for all bcc and hcp structures. The third set relies on the experimental Debye temperatures of the elements $\mathrm{Ta}(\mathrm{bcc})$ and $\mathrm{Ti}(\mathrm{hcp})$ [47]. These experimental values gave scaling factors of 0.58 for Ta (bcc) and 0.66 for Ti (hcp). The same scaling factors were used for the solid solutions of the bcc and hcp phases, respectively. For the fourth set we computed the Poisson ratios, 0.366 for Ta (bcc) and 0.319 for $\mathrm{Ti}$ (hcp), using the AEL elastic constants method implemented within AFLOW, which uses the algorithm described by de Jong et al. [49]. Then, the corresponding scaling factors from Eq. (8), 0.61 and 0.72 , were used for the solid solutions of the bcc and hcp lattices respectively. These four sets gave quite different Debye temperatures and vibrational energies but very similar excess vibrational energies for both lattices (see Supplement). Representative excess energies calculated using the generic scaling factors for the bcc and hcp phases for selected temperatures are shown in 
Fig. 4. These energies were again fitted to a sub-subregular model to retrieve the temperature dependence of the Redlich-Kister coefficients shown in Fig. 5 and table 2.

To compute the Gibbs energies for the bcc and hcp solutions (Eq. (2)), the Gibbs energies of the pure elements, ${ }^{0} \mathrm{G}_{\mathrm{Ta}}$ and ${ }^{0} \mathrm{G}_{\mathrm{Ti}}$, have to be considered for each phase. It is well known that the empirical lattice stabilities of the phase structures of pure elements (i.e. the energy differences between the stable phase and unstable phases), assessed, for example, by the Scientific Group Thermodata Europe (SGTE) database [50], are not consistent with first principles results for most of the transition metals $[51,52,53,54]$. According to the SGTE, $\Delta \mathrm{H}_{\mathrm{Ta}}{ }^{\text {bcc-hcp }}=-0.124 \mathrm{eV} / \mathrm{at}$, and $\Delta \mathrm{H}_{\mathrm{Ti}}{ }^{\text {hcp-bcc }}=-0.071$ eV/at. The corresponding values obtained by the DFT calculations are $\Delta \mathrm{H}_{\mathrm{Ta}}{ }^{\text {bcc-hcp }}=-0.278$ $\mathrm{eV} /$ at and $\Delta \mathrm{H}_{\mathrm{Ti}}^{\text {hcp-bcc }}=-0.11 \mathrm{eV} / \mathrm{at}$.

Fig. 6 shows the Gibbs energy curves for Ta-Ti solid solutions on the hcp and bcc lattices computed from Eq. (2), using the SGTE values for the pure elements with the excess energies computed as described above with DFT and AFLOW-AGL. Fig. 7 shows the corresponding Ta-Ti phase diagram for these energy curves computed by the Thermo-Calc software [55]. According to Fig. 6, two stable hcp phases appear at different stoichiometries at low temperatures. Moreover, the unexpected hcp phase near Ti concentration of 0.2 survives to temperatures above $882^{\circ} \mathrm{C}$. These features contradict the experimental data on the Ta-Ti system and are evidently incorrect. Fig. 7 illustrates this clear discrepancy between the computed phase diagram (solid lines) and the current experimentally assessed solvus (dashed line).

To overcome these obvious contradictions with the experimental data it is necessary to correct the lattice stabilities of the pure elements using the DFT calculated values, $\Delta \mathrm{H}_{\mathrm{Ta}}{ }^{\text {bcc-hcp }}=-0.278 \mathrm{eV} / \mathrm{at}$ for Ta and $\Delta \mathrm{H}_{\mathrm{Ti}}^{\text {hcp-bcc }}=-0.11 \mathrm{eV} / \mathrm{at}$ for Ti. This means that the Gibbs energy of hcp- Ta should be increased by $0.154 \mathrm{eV} /$ at compared to the SGTE value. For Ti, the known existence of a phase 
transition at $882^{\circ} \mathrm{C}$ allows us to derive a temperature dependent correction of $0.039-3.38 \cdot 10^{-5} \mathrm{~T} \mathrm{eV} / \mathrm{at}$. The calculations of the Gibbs energies (Fig. 8) and the phase diagram (Fig. 9) after these corrections exhibit a phase behavior much more in-line with the experimental data.

At room temperature, Fig. 8a, the Ti rich alloys have the lowest energy in the hcp phase and the Ta rich alloys have the lowest energy in the bcc phase. At this temperature, the convex hull of minimum energy is obtained by mixtures of these two phases. This agrees well with the known experimental phase diagram, where a wide phase-separation region exists at low temperatures. At $300^{\circ} \mathrm{C}$, Fig. $8 \mathrm{~b}$, the Gibbs energy of the hcp structure increases relative to the energy of the bcc structure. Moreover, the shape of the bcc energy curve dictates a complex convex hull at intermediate compositions. Mixing between the hcp and bcc structures is expected only for Ti rich alloys, and a miscibility gap in the bcc structure emerges at $15-58 \mathrm{at} \% \mathrm{Ti}$, with an intermediate stable bcc phase at 58-68at\% Ti. At $882^{\circ} \mathrm{C}$, just above the hcp to bcc phase transition of pure Ti (Fig. 8c), the Gibbs energy of the hcp structure continues to increase relative to that of the bcc phase, and the hcp structure becomes unstable for the entire range of compositions. This again agrees well with the known experimental phase diagram, where a bcc solid solution exists at all compositions at high temperatures, above the hcp-bcc phase transformation of Ti.

The calculated phase diagram, Fig. 9 , is qualitatively similar to the currently published experimental phase diagram of Fig. 1 at very low or very high temperatures. Yet at intermediate temperatures, the characteristics of the solvus between the phase separation regime and the solid solution region are markedly different. The main new feature of this calculated phase diagrams is a wide phase separation hump on the solvus that emerges at intermediate temperatures, below the interpolated solvus of the experimental diagram. It shows that the monotonic solvus of the experimental phase diagram should be replaced by a more complex structure that separates two narrow regions of stable bcc phases defined by monotectoid points. 
The computed phase diagram predicts a critical temperature at $\sim 500^{\circ} \mathrm{C}$ and $35 \mathrm{at} \% \mathrm{Ti}$, a monotectoid temperature at $\sim 230^{\circ} \mathrm{C}$ and miscibility gap for the two bec phases at $\sim 15-60$ at $\% \mathrm{Ti}$. The predicted critical temperature is very close to the lower limit of the temperature range of the currently known experimental phase diagram. The predicted monotectoid temperature is much below it. Observation of the miscibility gap in the bcc solid solution might thus be difficult due to slow diffusion and equilibration processes. However, the same structural features are well known for closely related systems of elements with the same crystallographic phases and similar electronic structures, e.g. TaZr, Ta-Hf, Cr-Ti, Mo-Ti, V-Ti and W-Ti [24]. In these systems the monotectoid temperatures range between $667^{\circ} \mathrm{C}$, in the $\mathrm{Cr}$-Ti system, and $1083^{\circ} \mathrm{C}$, in the Ta-Hf system, and the critical temperatures are between $850^{\circ} \mathrm{C}$, in the Mo-Ti and V-Ti systems, and $1780^{\circ} \mathrm{C}$ in the Ta-Zr system. These higher values explain why they were much easier to observe, while the same structural features in the Ta-Ti system remained hidden.

\section{Discussion and Conclusion}

Accurate description of phase diagrams is fundamental in materials science. It is especially essential in materials development for shape memory applications where the stability of the meta-stable phases in the system is crucial for the performance of potential devices. Moreover, this information is necessary for designing annealing processes and for the prediction of the aging processes which may affect the alloy homogeneity and, consequently, its local mechanical properties and corrosion resistance.

The most recently reported Ta-Ti binary phase diagram displays a complete bcc solid solution at high temperatures. At lower temperatures, below the solvus, this solid solution decomposes into a Tirich hcp phase and a Ta-rich bcc phase. The experimental investigation of the exact location of the solvus is quite difficult since it is located at relatively low temperatures, where diffusion is very slow. The experimental reports on this question are therefore equivocal. It was suggested that this 
ambiguity in various studies might be associated with contaminations or inadequate experimental quench rates [21].

In this study we used ab-initio calculations to investigate the Ta-Ti phase diagram and provide better estimates of the solvus location and structure. Similar methodologies of phase diagram prediction using ab-initio calculations have not been applied yet to the Ta-Ti system, but were used previously for various binary alloys and produced good agreement between the calculations and experimental observations $[56,57,58,59]$. In this research we utilize all-electron and pseudopotential DFT calculations to compute the ground state enthalpy for various ordered structures and for the SQS that mimic the bcc and hcp solid solutions. The positive formation enthalpies obtained for all of these structures show that no stoichiometric compounds are expected in the Ta-Ti system, as described in the known experimental data, and that phase separation between bcc-Ta and hcp-Ti is indeed expected at low temperatures. The excess enthalpy computations for the bcc solid solution show repulsive interactions between $\mathrm{Ta}$ and $\mathrm{Ti}$ for high Ta concentrations and attractive interactions for high Ti concentrations. For the hcp solid solution, the computed excess enthalpy shows strong attractive interactions. The excess Gibbs energies at finite temperatures were calculated via the Debye model with Debye temperatures obtained from the ab-initio calculations and the GIBBS model, employing the generic scaling factor corrections known for the hcp and bcc phases [46, 47]. Alternative estimates of these scaling factors do not alter the composition dependence of the excess energies, thus demonstrating that the assessment of the phase diagram is insensitive to the exact choice of the Poisson ratios.

It is known that the computational construction of phase diagrams is sensitive to the lattice stability of the constituent elements $[51,52,60,61]$, i.e. the relative stability of different structures of the same element. Various approaches have been proposed to mitigate the well-known discrepancy between empirical assessments and the results of DFT calculations of the lattice stability for elements where one of the relevant phases is unstable $[51,60,62]$. To examine the expression of this sensitivity in 
the current case, we constructed the Ta-Ti phase diagram using two data sets. For the first, we utilized the Gibbs energies of the pure elements directly from the SGTE database. In the second, we introduce a correction for the unstable phases of the pure elements (Ta-hcp and Ti-bcc) to obtain the same energy differences with respect to their stable phases as computed by DFT. This comparison clearly demonstrates that the DFT corrections for the pure elements are essential for an evaluation of the phase diagram that reasonably reconstructs the experimental phase diagram in the limits of very low temperatures, where phase separation is obtained, or high temperatures, where the existence of continuum solid solution is well established over all compositions. This correction is especially significant for the hcp alloys, where the excess enthalpy exhibits strong attractive interactions. The calculations also provide a new prediction for the transition from the solid solution to the phase separation in the phase diagram, which is characterized by a miscibility gap in the bcc phase region. This prediction refines the current experimental picture where the transition temperature decreases monotonically with increasing Ta content. The predicted miscibility gap has relatively low monotectoid and critical temperatures and has therefore been difficult to detect by experiments. The new computed phase diagram improves our understanding of the thermodynamics of the system and provides a more solid basis for both fundamental and applied research into Ta-Ti alloys. It should motivate more careful experiments to verify the characteristic features of the miscibility gap, the monotectoid and critical temperatures. An accurate description of these features is especially important for the development of shape memory applications, which are considerably affected by the stability of the meta-stable phases in the system. This information is also necessary for designing better thermal annealing processes and for the prediction of aging processes in these alloys, which may affect their homogeneity, local mechanical properties and corrosion resistance.

\section{Acknowledgements}

S.C. and C.T. acknowledge partial support by ONR-MURI under Contract N00014-13-1-0635, DOD-ONR (N00014-14-1-0526), and the Duke University Center for Materials Genomics. O.L. thanks the Duke 
University Center for Materials Genomics for its hospitality. The authors acknowledge the CRAY Corporation for computational assistance. 


\section{$\underline{\text { References }}$}

1. Y. L. Zhou, M. Niinomi, T. Akahori, M. Nakai, H. Fukui, Comparison of Various Properties between Titanium-Tantalum Alloy and Pure Titanium for Biomedical Applications, Materials Transactions 48-3 (2007) 380-384.

2. E. B. Taddei, V. A. R. Henriques, C. R. M. Silva and C. A. A. Cairo, Production of new titanium alloy for orthopedic implants, Materials Science and Engineering C 24 (2004) 683-687.

3. Y. L. Zhou, M. Niinomi, Ti-25Ta alloy with the best mechanical compatibility in Ti-Ta alloys for biomedical applications,Materials Science and Engineering C 29-3 (2009) 1061-1065.

4. E. Eisenbarth, D. Velten, M. Müller, R. Thull, J. Breme, Biocompatibility of $\beta$-stabilizing elements of titanium alloys, Biomaterials 25 (2004) 5705-5713.

5. Y. L. Zhou, M. Niinomi, Microstructures and mechanical properties of Ti-50 mass $\%$ Ta alloy for biomedical applications, J. Alloys and Compounds 466 (2008) 535-542.

6. Y. L. Zhou, M. Niinomi, T. Akahori, Effects of Ta content on Young's modulus and tensile properties of binary $\mathrm{Ti}-\mathrm{Ta}$ alloys for biomedical applications, Materials Science and Engineering A 371 (2004) 283-290.

7. S. Miyazaki, H.Y. Kim, P. J. S. Buenconsejo, Esomat 2009-8th European Symposium on Martensitic Transformations, (2009). Doi:10.1051/esomat/200901003.

8. X. H. Zheng, J. H. Sui, X. Zhang, Z. Y. Yang, H. B. Wang, X. H. Tian and W. Cai, Thermal stability and high-temperature shape memory effect of $\mathrm{Ti}-\mathrm{Ta}-\mathrm{Zr}$ alloy, Scripta Materialia 68 (2013) 1008-1011.

9. J. Ma, I. Karaman, R. D. Noebe, High temperature shape memory alloys, International Materials Reviews 55 (2010) 257-315.

10. P. J. S. Buenconsejo, H. Y. Kim, H. Hosoda, S. Miyazaki, Shape memory behavior of Ti-Ta and its potential as a high-temperature shape memory alloy, Acta Materialia 57 (2009) 1068-1077.

11. P. J. S. Buenconsejo, H. Y. Kim, S. Miyazaki, Effect of ternary alloying elements on the shape memory behavior of Ti-Ta alloys, Acta Materialia 57 (2009) 2509-2515.

12. P. J. S. Buenconsejo, H. Y. Kim, S. Miyazaki, Novel $\beta$-TiTaAl alloys with excellent cold workability and a stable high-temperature shape memory effect, Scripta Materialia 64 (2011) 1114-1117.

13. H. Y. Kim, T. Fukushima, P. John S. Buenconsejo, T. Nam, and S. Miyazaki, Martensitic transformation and shape memory properties of $\mathrm{Ti}-\mathrm{Ta}-\mathrm{Sn}$ high temperature shape memory alloys, Materials Science and Engineering A 528 (2011) 7238-7246.

14. J. L. Murray, Ta-Ti Binary Alloy Phase Diagrams, in: T.B. Massalski (ed), Binary Alloy Phase Diagrams second ed. Vol.3, 1990, p 3430.

15. D. S. Smith, The constitution of tantalum-titanium alloys, J. Inst. Met. 81 (1952) 73-76. 
16. D. J. Maykuth, H. R. Ogden and R. I. Jaffee, Titanium-Tungsten and Titanium-Tantalum Systems, Transactions of the American Institute of Mining and Metallurgical Engineers 197: 2 (1953) 231-237.

17. P. B. Budberg and K. I. Shakova, Phase Diagram of the Titanium-Tantalum System, Izv. Akad. Nauk SSSR Neorg. Mat., 3, No. 4, (1967) 656; TR:Inorg. Mater., 3, No. 4 (1967) 577.

18. J. L. Murray (Ed.), Phase Diagrams of Binary Titanium Alloys, ASM International, Metals Park, $\mathrm{OH}(1987)$, p. 183, 227.

19. L. Kaufman, Coupled thermochemical and phase diagram data for tantalum based binary alloys, Calphad, 15 (1991) 243-259.

20. E. Cresson (ed), COST 507 - Thermochemical database for light metal alloys. N. Saunders (1997) Ta-Ti system Vol 2, p. 293-296.

21. J. L. Murray, The Ta-Ti (Tantalum-Titanium) system, Bulletin of alloy phase diagrams 2-1 (1981) 62-66.

22. S. Curtarolo, W. Setyawan, G. L. W. Hart, M. Jahnatek, R. V. Chepulskii, R. H. Taylor, S. Wang, J. Xue, K. Yang, O. Levy, M. J. Mehl, H. T. Stokes, D. O. Demchenko, D. Morgan, AFLOW: An automatic framework for high-throughput materials discovery, Comp. Mat. Sci. 58 (2012) 218 226.

23. A. Zunger, S.-H. Wei, L. G. Ferreira, J. E. Bernard, Special quasirandom structures, Phys. Rev. Lett. 65 (1990) 353-356.

24. ASM Alloy Phase Diagram Database, P. Villars, editor-in-chief; H. Okamoto and K. Cenzual, section editors. ASM International, Materials Park, OH, 44073, USA 2006-2013 (http://www1.asminternational.org/asmenterprise/APD/default.aspx).

25. C. Jiang, C. Wolverton, J. Sofo, L. Q. Chen, Z.-K. Liu, First-principles study of binary bec alloys using special quasirandom structures, Phys. Rev. B 69 (2004) 214202:1-10.

26. D. Shin, R. Arróyave, Z.-K. Liu, A. Van de Walle, Thermodynamic properties of binary hcp solution phases from special quasirandom structures, Phys. Rev. B 74 (2006), 024204:1-13 and the erratum in Phys. Rev. B 76 (2007), 069901

27. G. Kresse and J. Furthmüller, Efficient iterative schemes for $a b$ initio total-energy calculations using a plane-wave basis set, Phys. Rev. B 54 (1996) 11169-11183.

28. C. E. Calderon, J. J. Plata, C. Toher, C. Oses, O. Levy, M. Fornari, A. Natan, M. J. Mehl, G. L. W. Hart, M. Buongiorno-Nardelli, S. Curtarolo, The AFLOW Standard for High-Throughput Materials Science Calculations, Comp. Mat. Sci. 108A (2015) 233-238.

29. G. Kresse, D. Joubert, From ultrasoft pseudopotentials to the projector augmented-wave method, Phys. Rev. B 59 (1999) 1758-1775.

30. J. P. Perdew, K. Burke, M. Ernzerhof, Generalized Gradient Approximation Made Simple, Phys. Rev. Lett. 77 (1996) 3865-3868. 
31. H. J. Monkhorst, J. D. Pack, Special points for Brillouin-zone integrations, Phys. Rev. B 13 (1976) 5188-5192.

32. S. Curtarolo, W. Setyawan, S. Wang, J. Xue, K. Yang, R. H. Taylor, L. J. Nelson, G. L. W. Hart, S. Sanvito, M. Buongiorno-Nardelli, N. Mingo, O. Levy, AFLOWLIB.ORG: A distributed materials properties repository from high-throughput $a b$ initio calculations, Comp. Mat. Sci. 58 (2012) 227-235.

33. R. H. Taylor, F. Rose, C. Toher, O. Levy, K. Yang, M. Buongiorno-Nardelli, S. Curtarolo, A RESTful API for exchanging materials data in the AFLOWLIB.org consortium, Comp. Mat. Sci. 93 (2014) 178-192.

34. K. Schwarz, P. Blaha, G. K. H. Madsen, Electronic structure calculations of solids using the WIEN2k package for material sciences, Comp. Phys. Commun. 147 (2002) 71-76.

35. S. Cottenier: Density Functional Theory and the family of (L)APW-methods: a step-by-step introduction (Instituut Voor Kern-en Stralingsfysica, K. U. Leuven, Belgium), 2002, ISBN 90807215-1-4 (http://www,wien2k.at/reg_user/textbooks).

36. J. P. Desclaux, Hartree Fock Slater self consistent field calculations, Comp. Phys. Commun. 1-3 (1970) 216-222.

37. D. D. Koelling, B. N. Harmon, A technique for relativistic spin-polarized calculations, J. Phys. C: Solid State. Phys. 10-16 (1977) 3107-3114.

38. L. D. Marks, Optimization notes, $(2004)<w w w . w i e n 2 k . a t / r e g \_u s e r / t e x t b o o k s />$.

39. L. D. Marks, Fixed-point optimization of atoms and density in DFT, J. Chem. Theory Comput. 9 (2013) 2786-2800.

40. Y. A. Chang and W. A. Oates, Materials thermodynamics, John Wiley \& sons inc., Hoboken New Jersey, 2010.

41. S. Stolen and T. Grand, Chemical thermodynamics of materials, John Wiley \& sons Ltd, west Sussex, England, 2003.

42. O. Redlich, A. T. Kister, Algebraic Representation of Thermodynamic Properties and the Classification of Solutions, Industr. Eng. Chem. 40 (1948) 345-348.

43. S. Bajaj, A. Landa. P. Söderlind, P. E. A. Turchi, R. Arróyave, The U-Ti system: Strengths and weaknesses of the CALPHAD method, J. Nuclear Materials 419 (2011)177-185.

44. J. L. Murray in T. B. Massalski (Ed.), Binary Alloy Phase Diagrams, second ed., vol. 3, ASM, Materials Park OH 44073, USA, 1990, pp 3488-3493.

45. M. A. Blanco, E. Francisco, V. Luaña, GIBBS: isothermal-isobaric thermodynamics of solids from energy curves using a quasi-harmonic Debye model, Comp. Phys. Comm. 158 (2004) 57 72.

46. V. L. Moruzzi, J. F. Janak, K. Schwarz, Calculated thermal properties of metals, Phys. Rev. B 37 (1988) 790-799. 
47. Q. Chen, B. Sundman, Calculation of Debye temperature for crystalline structures - a case study on Ti, Zr, and Hf, Acta Materialia 49 (2001) 947-961.

48. C. Toher, J. J. Plata, O. Levy, M. de Jong, M. Asta, M. Buongiorno-Nardelli, S. Curtarolo, Highthroughput computational screening of thermal conductivity, Debye temperature, and Grüneisen parameter using a quasiharmonic Debye model, Phys. Rev. B 90 (2014) 174107:1-14.

49. M. de Jong et al., Charting the complete elastic properties of inorganic crystalline compounds, Scientific Data 2, 150009 (2015).

50. SGTE Unary database, version 4.4, 20 july 2001, <http://www.sgte.org>.

51. P. J. Craievich, M. Weinert, J. M. Sanchez and R. E. Watson, Local stability of nonequilibrium phases, Phys. Rev. Lett. 72 (1994) 3076-3079.

52. Y. Wang, S. Curtarolo, C. Jiang, R. Arroyave, T. Wang, G. Ceder, L.-Q. Chen and Z.-K. Liu, Ab initio lattice stability in comparison with CALPHAD lattice stability, Calphad 28 (2004) 79-90.

53. S. Curtarolo, D. Morgan, G. Ceder, Accuracy of ab initio methods in predicting the crystal structures of metals: A review of 80 binary alloys, Calphad 29(3) (2005) 163-211.

54. M. H. F. Sluiter, Ab initio lattice stabilities of some elemental complex structures, Calphad 30 (2006) 357-366.

55. J.O. Andersson, T. Helander, L. Höglund, P.F. Shi, and B. Sundman, Thermo-Calc and DICTRA, Computational tools for materials science. Calphad 26 (2002) 273-312.

56. M. Asta, D. de Fontaine, M. van Schilfgaarde, First-principles study of phase stability of Ti-Al intermetallic compounds, J. Mater. Res. 8-10 (1993) 2554-2568.

57. D. Raabe, B. Sander, M. Friák, D. Ma, J. Neugebauer, Theory-guided bottom-up design of $\beta$ titanium alloys as biomaterials based on first principles calculations: Theory and experiments, Acta Materialia 55 (2007) 4475-4487.

58. N. Sodré, J. C. Garcia, L. V. C. Assali, P. G. Gonzales-Ormeño, P. Blaha, H. M. Petrilli, C. G. Schön, Intrinsic uncertainty on $a b$ initio phase diagram and compound formation energy calculations: BCC Mo-Fe as a test case. Phys Status Solidi B 250-1 (2013) 77-85.

59. Z.-K. Liu, First-principles calculations and CALPHAD modeling of thermodynamics, J. Phase Equilib. Diffus. 30 (2009) 517-534.

60. G. Grimvall, B. Magyari-Koppe, V. Ozolins, K. A. Persson, Lattice instabilities in metallic elements, Rev. Mod. Phys. 84 (2012) 945.

61. M. Palumbo et al., Thermodynamic modeling of crystalline unary phases, Phys. Status Solidi B 251 (2014) 14.

62. V. Ozolins, First-principles calculations of the free energies of unstable phases: the case of fcc W, Phys. Rev. Lett. 102 (2009) 065702. 


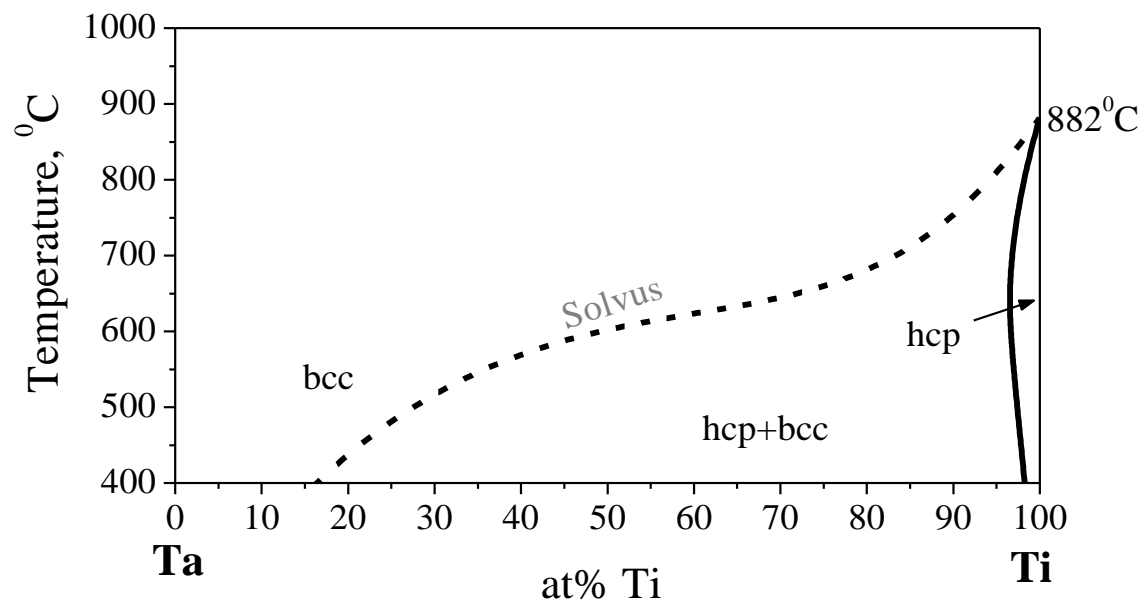

Fig. 1. Schematic Ta-Ti phase diagram assessed from experimental data [20].

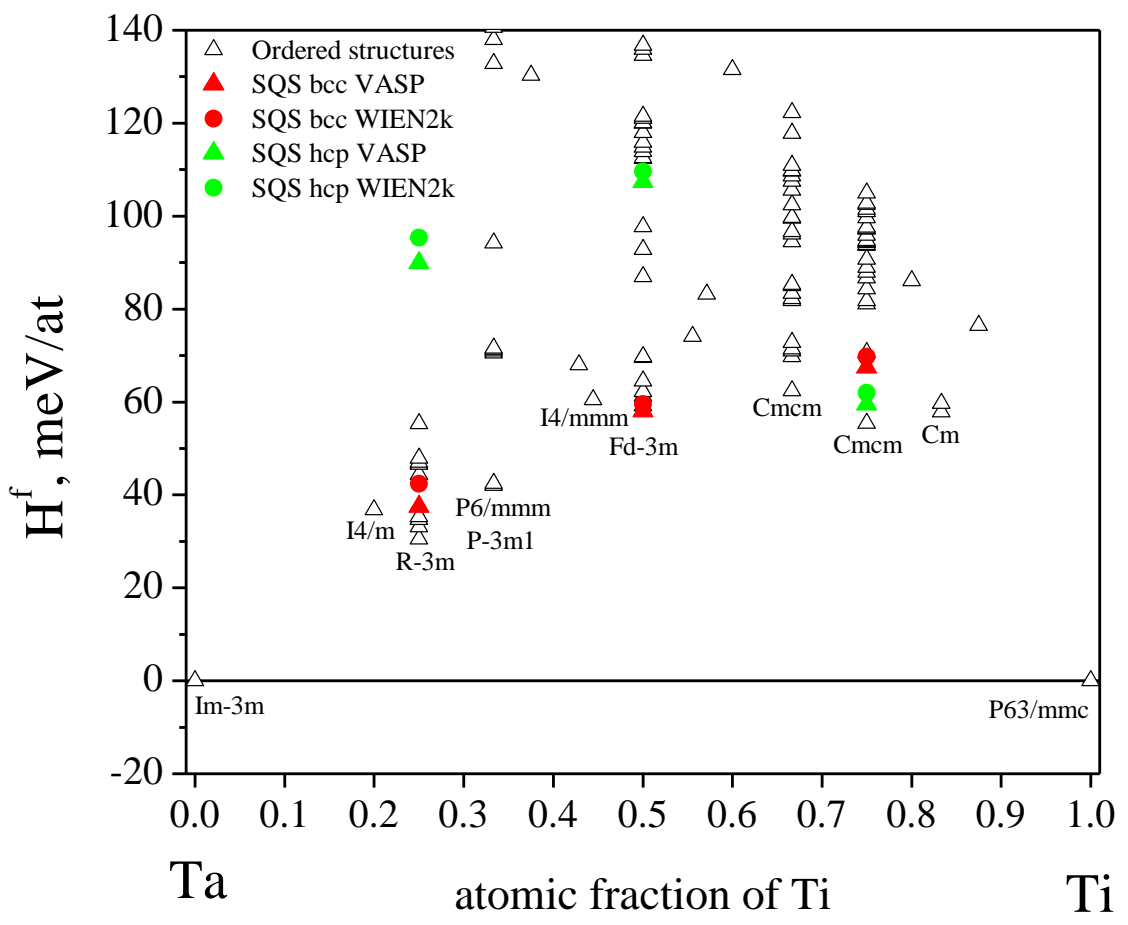

Fig. 2. Formation enthalpy of the lowest lying ordered compounds and the bcc- and hep- SQS computed with respect to bcc-Ta and hcp-Ti. 

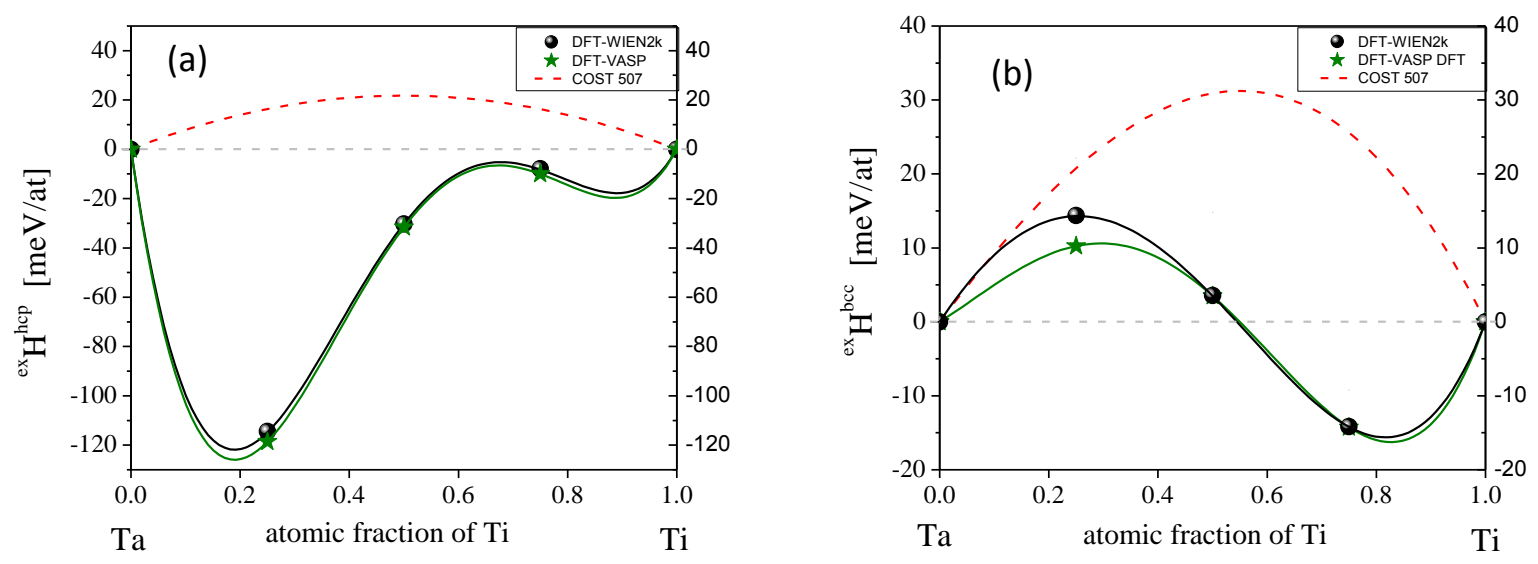

Fig. 3. Formation enthalpy as a function of composition for: (a) hcp Ta-Ti alloys with respect to the computed energy of hcp-Ta and hcp-Ti; (b) bcc Ta-Ti alloys with respect to the computed energy for bcc-Ta and bcc-Ti. The solid lines are the fits of the computed points to the sub-subregular model obtained from the VASP (green) and Wien2k (black) calculations. The red dash lines represent an assessment based on experimental data for hcp and bcc solid solutions [20].
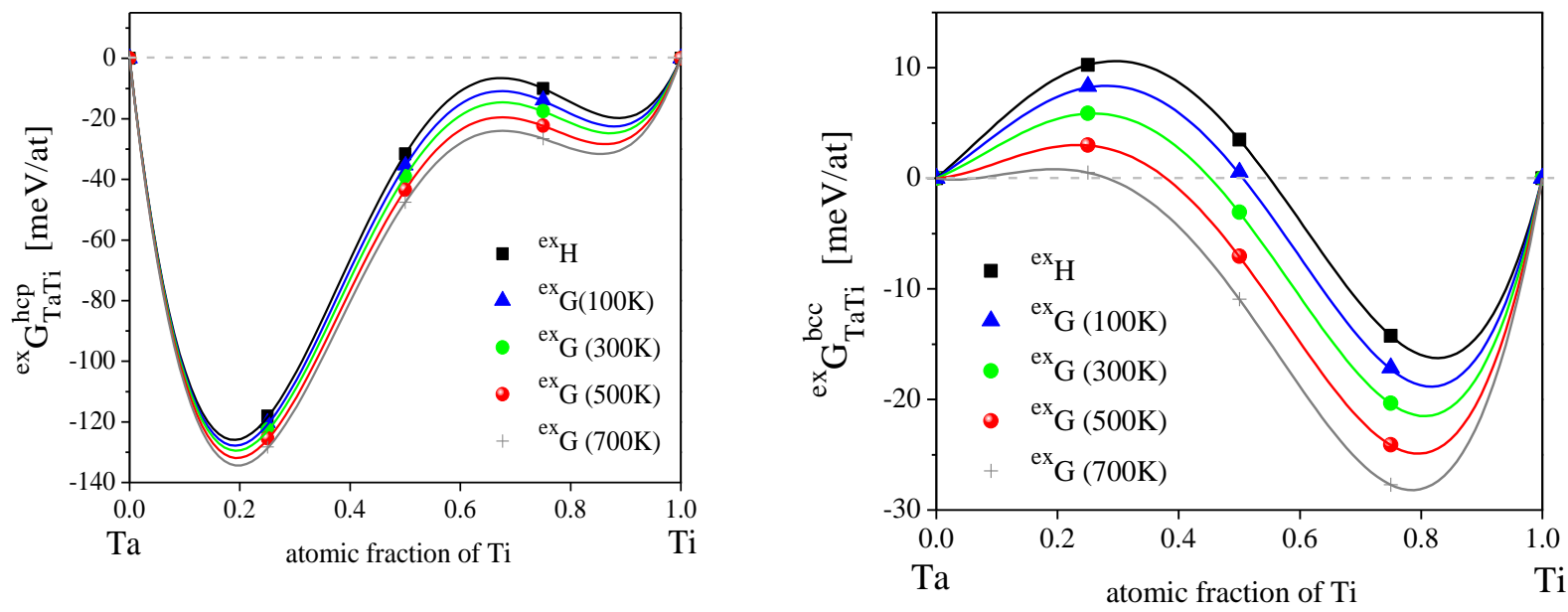

Fig. 4. Excess energies as a function of composition and temperature for hcp and bcc solid solutions in Ta-Ti alloys. The Debye temperatures for the selected compositions were computed from the DFT results by the GIBBS model [45, 48] with the generic scaling factors of 0.617 [46] and 0.7 [47] for the bcc and hcp structures, respectively. The solid lines represent the fits of the computed points to a sub-subregular model. 

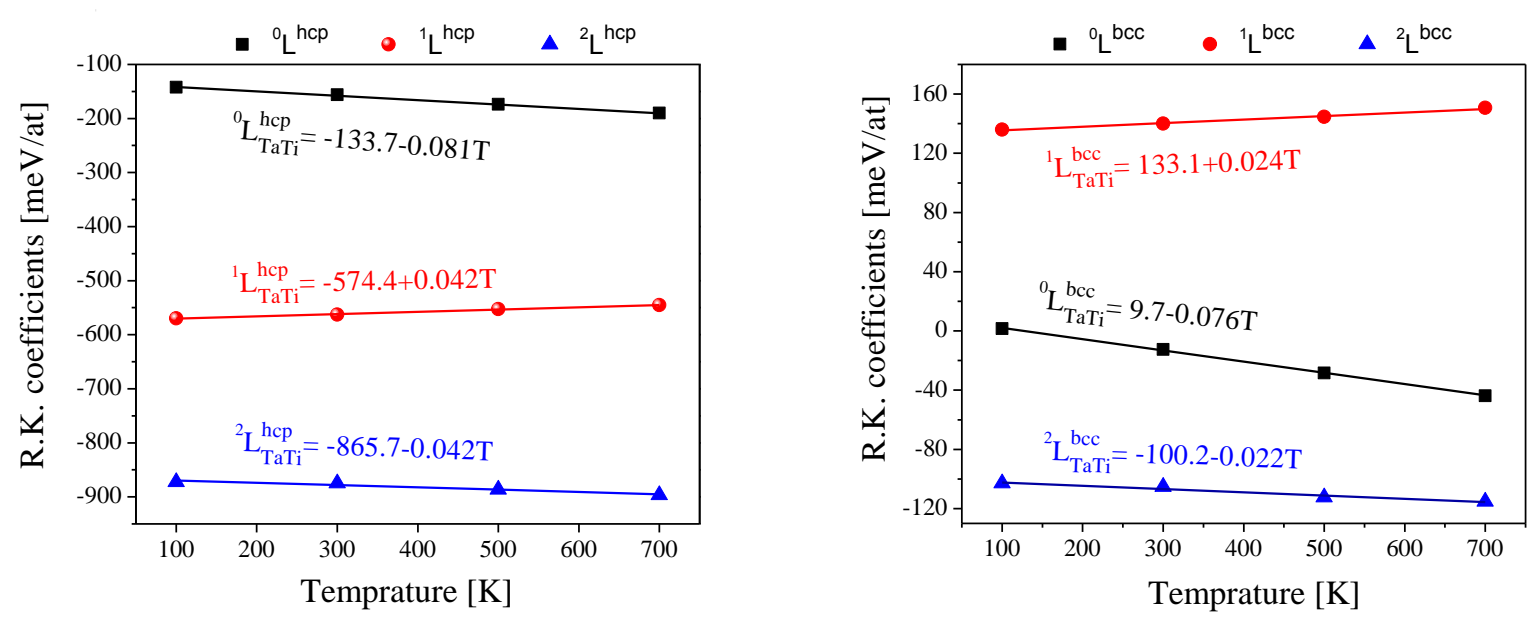

Fig. 5. The temperature dependent Redlich-Kister coefficients of the excess energies for the hcp and bcc phases in the Ta-Ti system.
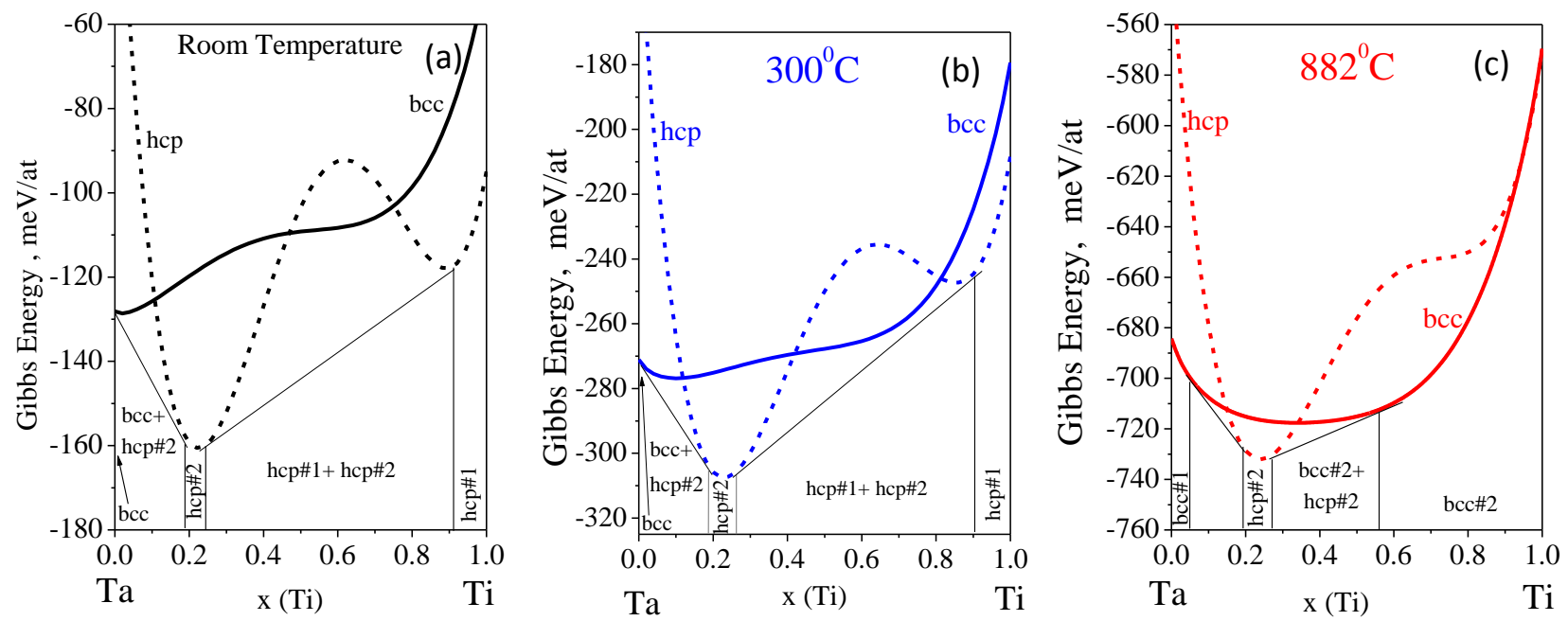

Fig. 6. The calculated Gibbs energy curves for the bcc and hcp structures of Ta-Ti alloys as obtained using the SGTE values for the pure elements and the computed excess energies. The curves were calculated at: (a) room temperature, (b) $300^{\circ} \mathrm{C}$ and (c) $882^{\circ} \mathrm{C}$. The dashed and the solid curves denote the energies of the hcp and the bcc solid solutions, respectively. The tangent lines mark the stable phase convex hull of the system. 


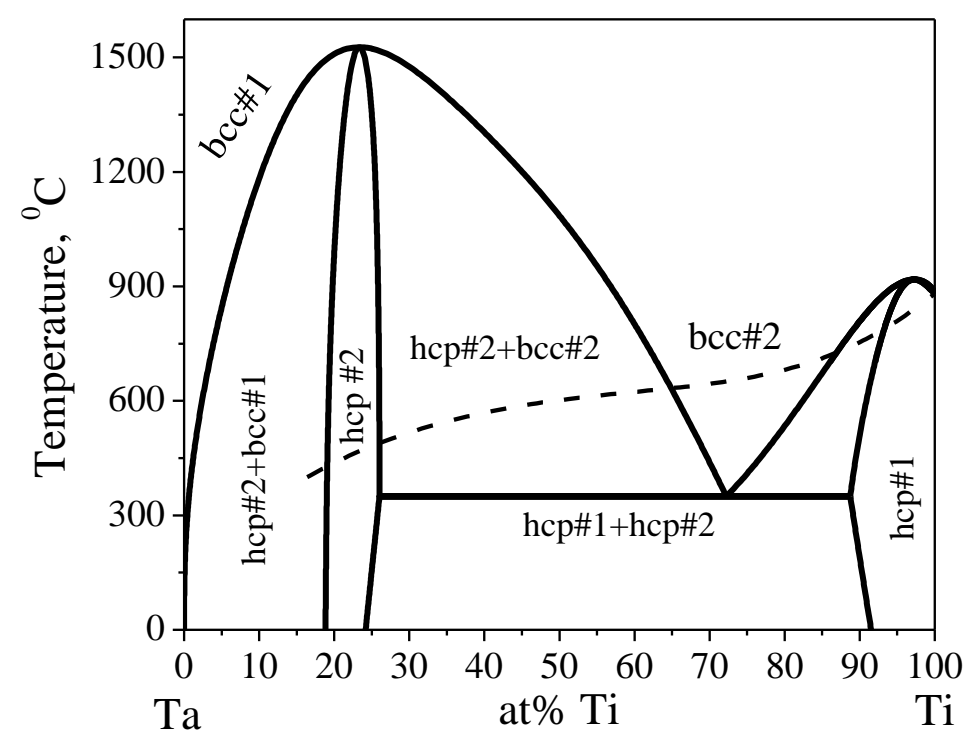

Fig 7. The calculated Ta-Ti phase diagram derived from the energy curves of Fig.6. The dashed line represents the monotonic solvus of the experimental phase diagram.
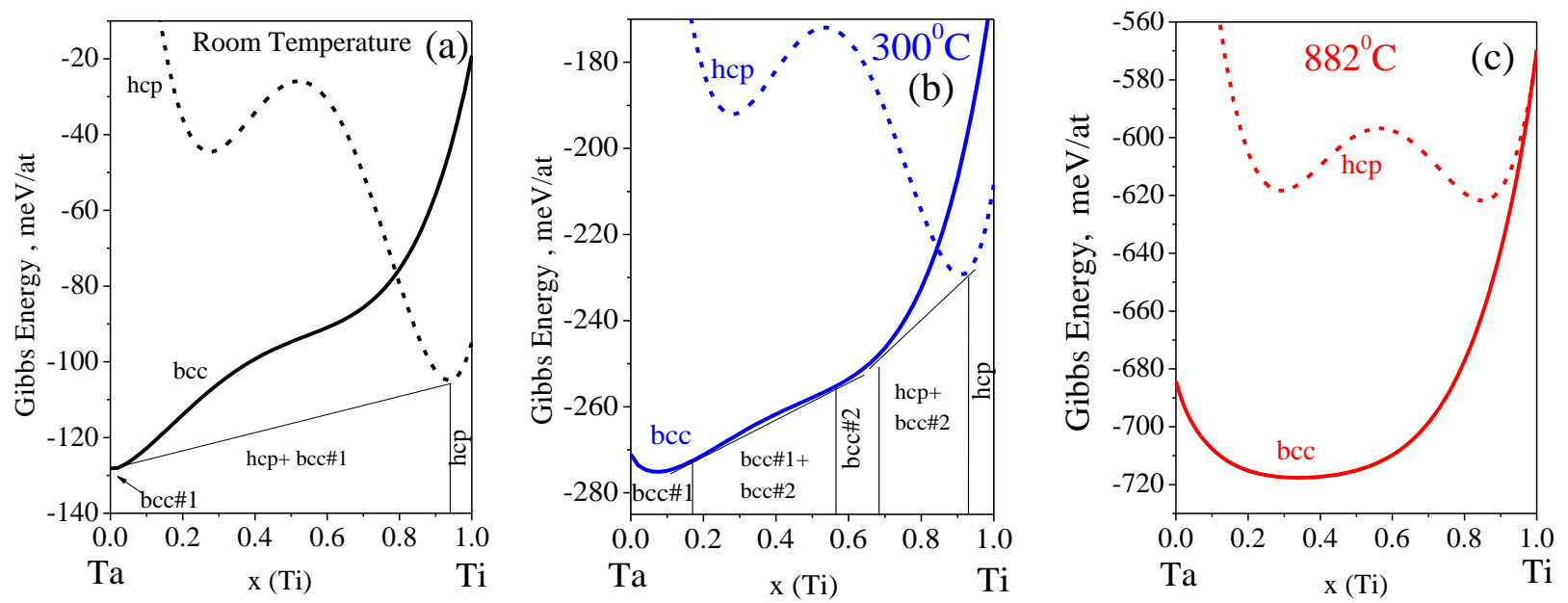

Fig. 8. The calculated Gibbs energy curves for the bcc and hcp structures of Ta-Ti alloys as obtained with the DFT-guided corrections for the Gibbs energies of the pure elements. The curves are calculated at: (a) room temperature, (b) $300^{\circ} \mathrm{C}$ and (c) $882^{\circ} \mathrm{C}$. The dash and the solid curves represent the energies of the hcp and the bcc solid solutions, respectively. The tangent lines mark the stable phase convex hull of the system. 


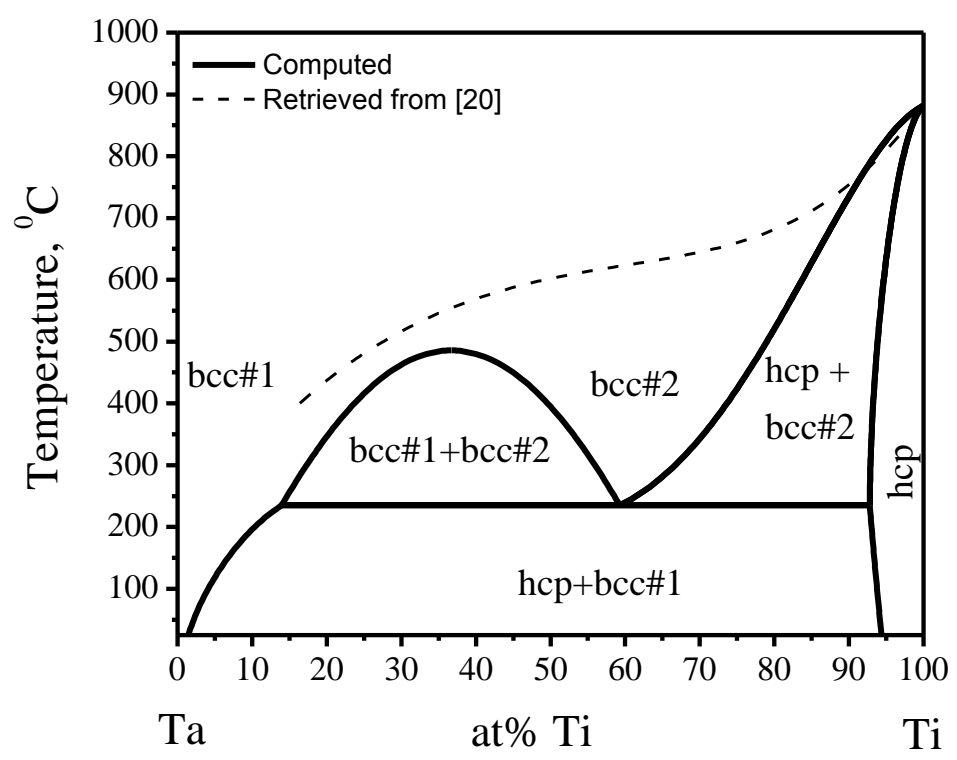

Fig 9. The calculated Ta-Ti phase diagram, based on ab-initio evaluation of the excess Gibbs energy, including corrections of the Gibbs energies of the pure elements. The dashed line represents the monotonic solvus of the experimental phase diagram. 
Table 1: The computed Bulk moduli, specific volumes and representative Debye temperatures calculated using the generic scaling factors for the selected structures.

\begin{tabular}{|c|c|c|c|c|c|c|c|c|}
\hline \multirow{3}{*}{ Phase } & \multirow{3}{*}{ Composition } & \multicolumn{7}{|c|}{$A b$-initio results } \\
\hline & & \multirow{2}{*}{$\begin{array}{c}\mathrm{V} \\
{\left[\AA^{3} / \text { atom }\right]}\end{array}$} & \multicolumn{3}{|c|}{$\mathrm{B}_{\mathrm{T}}[\mathrm{GPa}]$} & \multicolumn{3}{|c|}{$\theta_{\mathrm{D}}[\mathrm{K}]$} \\
\hline & & & $0 \mathrm{~K}$ & $300 \mathrm{~K}$ & $700 \mathrm{~K}$ & $0 \mathrm{~K}$ & $300 \mathrm{~K}$ & $700 \mathrm{~K}$ \\
\hline \multirow{5}{*}{ bcc } & $\mathrm{Ti}$ & 17.09 & 104 & 101 & 97 & 337 & 333 & 326 \\
\hline & $\mathrm{Ta}-75 \mathrm{at} \% \mathrm{Ti}$ & 17.45 & 127 & 124 & 117 & 288 & 285 & 279 \\
\hline & $\mathrm{Ta}-50 \mathrm{at} \% \mathrm{Ti}$ & 17.74 & 149 & 146 & 139 & 262 & 260 & 256 \\
\hline & $\mathrm{Ta}-25 \mathrm{at} \% \mathrm{Ti}$ & 18.02 & 170 & 167 & 161 & 247 & 246 & 243 \\
\hline & $\mathrm{Ta}$ & 18.29 & 195 & 191 & 185 & 240 & 239 & 236 \\
\hline
\end{tabular}

\begin{tabular}{|c|c|c|c|c|c|c|c|c|}
\hline \multirow{5}{*}{ hcp } & Ti & 17.25 & 110 & 107 & 99 & 392 & 388 & 378 \\
\cline { 2 - 9 } & Ta -75at\% Ti & 17.52 & 127 & 124 & 117 & 325 & 322 & 315 \\
\cline { 2 - 9 } & $\mathrm{Ta}-50 \mathrm{at} \% \mathrm{Ti}$ & 18.01 & 148 & 144 & 138 & 296 & 293 & 288 \\
\cline { 2 - 9 } & $\mathrm{Ta}-25 \mathrm{at} \% \mathrm{Ti}$ & 18.58 & 168 & 164 & 157 & 278 & 276 & 271 \\
\cline { 2 - 9 } & $\mathrm{Ta}$ & 18.74 & 188 & 184 & 178 & 267 & 265 & 262 \\
\hline
\end{tabular}

Table 2: The Redlich-Kister coefficients for the bcc and hcp phases of TaTi alloys. The experimental values correspond to a simpler, temperature independent, model.

\begin{tabular}{|l|l|l|l|l|}
\hline & Lattice & ${ }^{0} \mathrm{~L}[\mathrm{meV} / \mathrm{at}]$ & ${ }^{1} \mathrm{~L}[\mathrm{meV} / \mathrm{at}]$ & ${ }^{2} \mathrm{~L}[\mathrm{meV} / \mathrm{at}]$ \\
\hline $\begin{array}{l}\text { Ab-initio } \text { and GIBBS } \\
\text { model (current work) }\end{array}$ & $\mathrm{Bcc}$ & $9.7-0.076 \mathrm{~T}$ & $133.1+0.024 \mathrm{~T}$ & $-100.2-0.022 \mathrm{~T}$ \\
\cline { 2 - 5 } & $\mathrm{Hcp}$ & $-133.7-0.081 \mathrm{~T}$ & $-574.4+0.042 \mathrm{~T}$ & $-865.7-0.042 \mathrm{~T}$ \\
\hline $\begin{array}{l}\text { Evaluated from experimental } \\
\text { data [20] }\end{array}$ & Bcc & 123.6 & -25.75 & -- \\
\cline { 2 - 6 } & Hcp & 87 & -- & -- \\
\hline
\end{tabular}




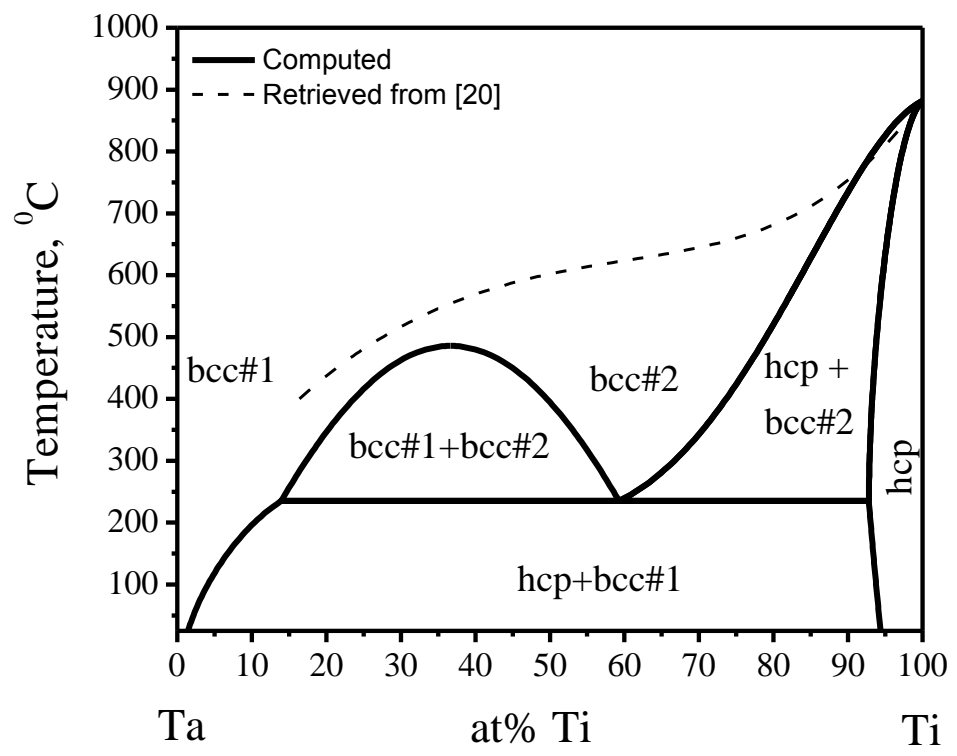

The calculated Ta-Ti phase diagram, based on ab-initio evaluation of the excess Gibbs energy, including corrections of the Gibbs energies of the pure elements. The dashed line represents the monotonic solvus of the experimental phase diagram. 